\title{
Modernised GPS: Phase-only Integer Ambiguity Estimability in the Presence of Ionospheric Delays
}

\author{
D. Odijk and P.J.G. Teunissen \\ Department of Mathematical Geodesy and Positioning \\ Delft University of Technology \\ Thijsseweg 11 \\ 2629 JA Delft, The Netherlands \\ Fax: ++ 31152783711
}

\begin{abstract}
In GPS data processing it is not uncommon to eliminate the presence of ionospheric delays by taking certain linear combinations of the carrier phase data. This approach is in fact equivalent with a processing of the original, not combined, $L_{1}$ and $L_{2}$ phase observations in which the ionospheric delays are modelled as unknown parameters. When using phase data only, the presence of the unknown ionospheric delays produces a rank defect in the model of observation equations. Eliminating this rank defect leads to the conclusion that not all of the original $L_{1}$ and $L_{2}$ ambiguities can be estimated as integers, but only a certain linear combination of them. In this contribution it is investigated how this situation changes when triple-frequency, phase-only data of modernised GPS are used. We identify the integer rank defects, show which integer ambiguity combinations are estimable and determine their corresponding ambiguity success-rates.
\end{abstract}

Keywords. GPS, triple-frequency, ambiguity resolution, rank-defect integer least-squares, ionosphere

\section{Introduction}

Ambiguity resolution is the process of resolving the unknown cycle ambiguities of double difference (DD) carrier phase data as integers. Apart from the current Global Positioning System (GPS) models, carrier phase ambiguity resolution also applies to the future modernized GPS and the future European Galileo GNSS. An overview of GNSS models, together with their applications in surveying, navigation, geodesy and geophysics, can be found in textbooks such as [Hofmann-Wellenhof et al., 1997], [Leick, 1995], [Parkinson and Spilker, 1996], [Strang and Borre, 1997] and [Teunissen and Kleusberg, 1998].

In this contribution we study the integer estimability of the ambiguities in case phase-only data is used for long baselines. The potential advantage of using carrier phase data only is that ambiguity resolution will be freed from the effects of code (or pseudorange) multipath. Fast phase-only ambiguity resolution has been shown possible for short baselines, see e.g. [Teunissen, 1995], [Tiberius and de Jonge, 1995] and [Teunissen et al., 1997]. For long baselines the situation becomes more problematic due to the presence of the ionospheric delays. This is certainly true for the present dual frequency GPS system. It is therefore of interest to study whether the inclusion of a third frequency, as will be the case with modernised GPS, allows one to improve the performance of phase-only ambiguity resolution significantly.

This contribution is organized as follows. In section 2 we present briefly some theory for tackling rank defect ambiguity resolution problems. Although this theory is of interest in its own right, it is particularly relevant for solving the phase-only ambiguity resolution problem for long baselines. By introducing a suitable ambiguity transformation, we identify in section 3 which integer ambiguities are estimable and which are not. We also show how the real-valued parameters are affected by the transformation. The results of section 3 are then finally used in section 4 to compute the success-rates of the estimable integer ambiguities.

\section{Rank defect ambiguity resolution}

\subsection{The GPS model}

As our point of departure we will take the following system of linear(ized) observation equations

$$
y=A a+B b+e
$$

where $y$ is the given GPS data vector of order $m$, $a$ and $b$ are the unknown parameter vectors respectively of order $n$ and $p$, and where $e$ is the noise vector. In principle all the GPS models can be cast in 
this frame of observation equations. The data vector $y$ may consist of the 'observed minus computed' single-, dual- or triple frequency double-difference (DD) phase and/or pseudorange (code) observations accumulated over all observation epochs. The entries of vector $a$ are then the DD carrier phase ambiguities, expressed in units of cycles rather than range. They are known to be integers, $a \in Z^{n}$. The entries of the vector $b$ will consist of the remaining unknown parameters, such as for instance baseline components (coordinates) and possibly atmospheric delay parameters (troposphere, ionosphere). They are known to be real- valued, $b \in R^{p}$.

The procedure which is usually followed for solving the GPS model (1), can be divided into three steps. In the first step one simply disregards the integer constraints $a \in Z^{n}$ on the ambiguities and performs a standard least-squares adjustment. As a result one obtains the (real-valued) estimates of $a$ and $b$, together with their variance-covariance (vc-) matrix

$$
\left[\begin{array}{c}
\hat{a} \\
\hat{b}
\end{array}\right],\left[\begin{array}{cc}
Q_{\hat{a}} & Q_{\hat{a} \hat{b}} \\
Q_{\hat{b} \hat{a}} & Q_{\hat{b}}
\end{array}\right]
$$

This solution is referred to as the 'float' solution. In the second step the 'float' ambiguity estimate $\hat{a}$ is used to compute the corresponding integer leastsquares ambiguity estimate

$$
\check{a}=\arg \min _{a \in Z^{n}}\|\hat{a}-a\|_{Q_{\hat{a}}}^{2}
$$

with $\|\cdot\|_{Q_{\hat{a}}}^{2}=(.)^{T} Q_{\hat{a}}^{-1}($.$) and where Q_{\hat{a}}^{-1}=$ $\bar{A}^{T} Q_{y}^{-1} \bar{A}$ with $\bar{A}=P_{B}^{\perp} A, P_{B}^{\perp}=I_{p}-$ $B\left(B^{T} Q_{y}^{-1} B\right)^{-1} B^{T} Q_{y}^{-1}$ and $Q_{y}$ the vc-matrix of the observables.

Once the integer ambiguities are computed, they are used in the third step to finally correct the 'float' estimate of $b$. As a result one obtains the 'fixed' solution

$$
\check{b}=\hat{b}-Q_{\hat{b} \hat{a}} Q_{\hat{a}}^{-1}(\hat{a}-\check{a})
$$

In the present contribution we will show how the above procedure needs to be modified in case one wants to solve for the triple-frequency, phase-only, rank-defect GPS model.

\subsection{Eliminating the rank defect}

Let us assume that the design matrix $(A, B)$ of (1) has a rank defect of $r>0$ and that this defect manifests itself completely in the $n \times n$ reduced normal matrix $\bar{A}^{T} Q_{y}^{-1} \bar{A}$. The null-space of the reduced normal matrix will then be non-empty and the 'float' solution $\hat{a}$ fails to be unique. If the columns of the $n \times r$ matrix $X$ span the null space of the reduced normal matrix, then any of the vectors $\hat{a}+X x$, with arbitrary $x \in R^{r}$, will constitute a 'float' least-squares solution. It now depends on the signature of the null space whether or not the non-uniqueness of the 'float' solution carries over to the integer ambiguity solution. This non-uniqueness is not carried over in case $X x$ fails to be an integer vector. However, the integer solution will become non-unique as well, in case the null space can be spanned by integer vectors. It is this latter case which applies to the long baseline, phase-only, modernised GPS model.

In order to handle the above type of nonuniqueness in the integer ambiguity solution, we may proceed as follows. Let the $n \times n$ matrix $Z=$ $\left(Z_{1}, Z_{2}\right)$ be an admissible ambiguity transformation (i.e. it is volume preserving and it has integer entries only), set $\hat{a}=Z \hat{z}$ and $a=Z z$, and assume that the integer columns of $Z_{1}$ span the null-space of the reduced normal matrix. Then

$$
\begin{gathered}
\|\hat{a}-a\|_{Q_{\hat{a}}}^{2}=\left(\hat{z}_{2}-z_{2}\right)^{T} Z_{2}^{T} \bar{A}^{T} Q_{y}^{-1} \bar{A} Z_{2}\left(\hat{z}_{2}-z_{2}\right) \\
\text { since } \bar{A} Z_{1}=0
\end{gathered}
$$

The $(n-r) \times(n-r)$ matrix $Z_{2}^{T} \bar{A}^{T} Q_{y}^{-1} \bar{A} Z_{2}$ is now of full rank. We may therefore minimize the above quadratic form as function of $z_{2} \in Z^{n-r}$ using standard techniques again. If we denote the solution as $\check{z}_{2}$, the non- unique, but integer least-squares solution of the original ambiguities becomes

$$
\check{a}=Z_{1} z_{1}+Z_{2} \check{z}_{2} \text { with arbitrary } z_{1} \in Z^{r}
$$

\subsection{The ambiguity success-rate}

Once the rank defect has been eliminated one can think of resolving the transformed integer ambiguities $z_{2}$. How well the transformed ambiguities can be resolved is measured by their ambiguity success-rate. The ambiguity success-rate is defined as the probability of correct integer least-squares estimation. It is given as

$$
P\left(\check{z}_{2}=z_{2}\right)=\int_{S_{z_{2}}} p_{\hat{z}_{2}}(x) d x, z_{2} \in Z^{n-r}
$$

with the integer least-squares estimator $\check{z}_{2}=$ $\arg \min _{z \in Z^{n-r}} \quad\left\|\quad \hat{z}_{2}-z \quad\right\|_{Q_{\hat{z}_{2}}}^{2}, Q_{\hat{z}_{2}}=$ $\left(Z_{2}^{T} \bar{A}^{T} Q_{y}^{-1} \bar{A} Z_{2}\right)^{-1}$, and the pull-in region $S_{z_{2}}=$ $\left\{x \in R^{n-r} \mid\left\|x-z_{2}\right\|_{Q_{\hat{z}_{2}}}^{2} \leq\|x-z\|_{Q_{\hat{z}_{2}}}^{2}\right.$ ,$\left.\forall z \in Z^{n-r}\right\}$ as the region of integration. Since we assume the data to be normally distributed, the pdf of 
the 'float' solution $\hat{z}_{2}$ is given as

$$
p_{\hat{z}_{2}}(x)=\frac{\exp \left\{-\frac{1}{2}\left\|x-z_{2}\right\|_{Q_{\hat{z}_{2}}}^{2}\right\}}{\sqrt{\operatorname{det}\left(Q_{\hat{z}_{2}}\right)}(2 \pi)^{\frac{1}{2}(n-r)}}
$$

Different ways of evaluating the above multi-variate integral have been given in [Teunissen, 1998]. In the present contribution we will make use of the very easy-to-compute lower bound

$$
\Pi_{i=1}^{n-r}\left(2 \Phi\left(\frac{1}{2 \sigma_{i \mid I}}\right)-1\right) \leq P\left(\check{z}_{2}=z_{2}\right)
$$

with

$$
\Phi(x)=\int_{-\infty}^{x} \frac{1}{\sqrt{2 \pi}} \exp \left\{-\frac{1}{2} v^{2}\right\} d v
$$

and where the $\sigma_{i \mid I}, i=1, \ldots,(n-r)$, denote the sequential conditional standard deviations of the decorrelated ambiguities $\hat{z}_{2}$. The validity of the lower bound is a consequence of the success-rate's optimality property of integer least-squares as proven in [Teunissen, 1999] and it is presently the sharpest lower bound available for (7), see e.g. [Thomsen, 2000].

\subsection{The non-uniqueness of $\breve{b}$}

The fact that the integer ambiguities can not be estimated uniquely may also have its affect on the 'fixed' solution $\check{b}$. To see this, consider $\bar{A} Z_{1}=0$, with $\bar{A}=P_{B}^{\perp} A$. From these two equations it follows that the range space of $A Z_{1}$ is a subspace of the range space of $B$. Hence, there exists a $p \times r$ matrix $Y_{1}$ such that the null space of $(A, B)$ is spanned by the $r$ columns of the matrix $\left(Z_{1}^{T}, Y_{1}^{T}\right)^{T}$. These $r$ columns can be complemented to obtain the one-to-one parameter transformation

$$
\left[\begin{array}{l}
a \\
b
\end{array}\right]=\left[\begin{array}{ccc}
Z_{1} & Z_{2} & 0 \\
Y_{1} & 0 & I_{p}
\end{array}\right]\left[\begin{array}{c}
z_{1} \\
z_{2} \\
b^{\prime}
\end{array}\right]
$$

With this parameter transformation, the observation equations become $y=A Z_{2} z_{2}+B b^{\prime}+e$. These equations are now of full rank. To see whether or not the non- uniqueness in the integer ambiguities affects the 'fixed' solution of $b^{\prime}$, we need the inverse of (9). It reads

$$
\left[\begin{array}{c}
z \\
b^{\prime}
\end{array}\right]=\left[\begin{array}{lc}
\left(Z_{1}, Z_{2}\right)^{-1} & 0 \\
-\left(Y_{1}, 0\right)\left(Z_{1}, Z_{2}\right)^{-1} & I_{p}
\end{array}\right]\left[\begin{array}{l}
a \\
b
\end{array}\right]
$$

This shows that the entries of $b^{\prime}$ will equal their counterparts of $b$ when the corresponding rows of matrix $Y_{1}$ equal zero. These entries of $b^{\prime}$ are therefore not affected by the non-uniqueness of the integer ambiguities.

\section{The long baseline, phase-only mod- ernised GPS model}

In this section we will consider the long baseline, phase-only modernised GPS model. Since the emphasis is on long baselines, the ionospheric delays will be included as unknown parameters in the model. The number of satellites tracked will be denoted by $s$. For a single epoch $i(i=1, \ldots k)$, a single frequency $f_{j}(j=1,2,3)$ and a stationary baseline, the linearized system of $s-1$ DD carrier phase observation equations reads

$$
\phi_{j}(i)=\lambda_{j} a_{j}-\mu_{j} I(i)+G(i) g
$$

with $\phi_{j}(i)$ the vector of $s-1 \mathrm{DD}$ carrier phase data on frequency $f_{j}, a_{j}$ the unknown time invariant vector of the $s-1$ integer ambiguities of frequency $f_{j}$, $I(i)$ the vector of the $s-1$ unknown DD ionospheric delays and $g$ the vector of the three unknown baseline components. The $(s-1) \times 3$ matrix $G(i)$ captures the relative receiver-satellite geometry at epoch $i, \lambda_{j}$ for $j=1,2,3$ denote the known wavelengths of modernised GPS and the $\mu_{j}$ are equal to $\mu_{1}=1$, $\mu_{2}=\left(\lambda_{2} / \lambda_{1}\right)^{2}, \mu_{3}=\left(\lambda_{3} / \lambda_{1}\right)^{2}$. The wavelength ratios of modernised GPS are given as $\lambda_{2} / \lambda_{1}=77 / 60$, $\lambda_{3} / \lambda_{1}=154 / 115$ and $\lambda_{3} / \lambda_{2}=24 / 23$. Since it will be assumed that at least 4 satellites are tracked, we have $s \geq 4$.

The above system of observation equations will have a rank defect due to the absence of code data and the presence of the unknown ionospheric delays. The presence of the ionospheric delays requires the use of at least two frequencies, while the absence of the code data requires that at least two epochs of data are used. However even if these requirements are met, a rank defect of $s-1$ will remain. We will now show how the theory of the previous section works out for the above model (11). The proofs are omitted due to space limitations.

For the admissible ambiguity transformation $z=$ $Z^{-1} a$ we obtain

$$
\begin{aligned}
& z_{1}=90 a_{1}-67 a_{2}-3 a_{3} \\
& z_{2}=77 a_{1}-60 a_{2} \\
& z_{3}=24 a_{2}-23 a_{3}
\end{aligned}
$$

Of these transformed integer ambiguities, $z_{2}$ and $z_{3}$ are estimable, whereas the $s-1$ ambiguities of $z_{1}$ are not. The latter ambiguities will therefore not appear in the reparametrized carrier phase observation equations. This takes care of the rank defect.

For the transformed real-valued parameters $b^{\prime}=$ 
$b-\left(Y_{1}, 0\right) Z^{-1} a$ we obtain

$$
\begin{array}{ll}
I^{\prime}(i) & =I(i)+60 \times 23 \lambda_{1} z_{1} \\
g^{\prime} & =g
\end{array}
$$

This shows that the baseline is not affected by the elimination of the rank defect, but that the ionospheric delays are affected. The ionospheric delays become biased due to the presence of the undetermined ambiguities $z_{1}$. But since the bias is time invariant, the time variability of the ionospheric delays will remain unbiased estimable. The observation that the baseline remains unbiased estimable is important, since it implies that, in principle, phase-only ambiguity resolution is applicable for long baselines as well. But note that the elimination of the rank defect has effectively reduced the triple-frequency system back to a dual frequency system. In the next section we will show how well one can expect such a system to perform.

\section{The triple-frequency, phase-only success-rates}

We will now show how well one can expect ambiguity resolution to perform over long baselines using phase data only. We therefore computed the successrates of the integer estimable ambiguities

$$
\begin{aligned}
& z_{2}=77 a_{1}-60 a_{2} \\
& z_{3}=24 a_{2}-23 a_{3}
\end{aligned}
$$

The standard deviation of the undifferenced carrier phase data was set at the value of $2 \mathrm{~mm}$ and the sampling interval was chosen equal to 10 seconds. We refrained from taking shorter intervals, because of the potential presence of time correlation. Choosing shorter intervals, with a corresponding shortening in time of successful ambiguity resolution, is possible when it can be assured that the data remains free of time correlation. The receiver-satellite geometry used is shown in the skyplot of figure 1. It covers a period of 20 minutes. In order to show the dependence of the success-rate on the number of satellites tracked, the following three scenarios were taken: $s=8, s=6$ (PRNs 1,4,5,6,9,24) and $s=4$ (PRNs $1,4,5,6)$.

The corresponding success-rates are shown in figure 2. In figure $2 \mathrm{a}$ the success-rates are shown in case one aims at resolving all the integer estimable ambiguities of (14). As the figure shows, the ambiguity success-rate gets larger when the observational period gets larger or when the number of satellites tracked gets larger. The figure also shows however,

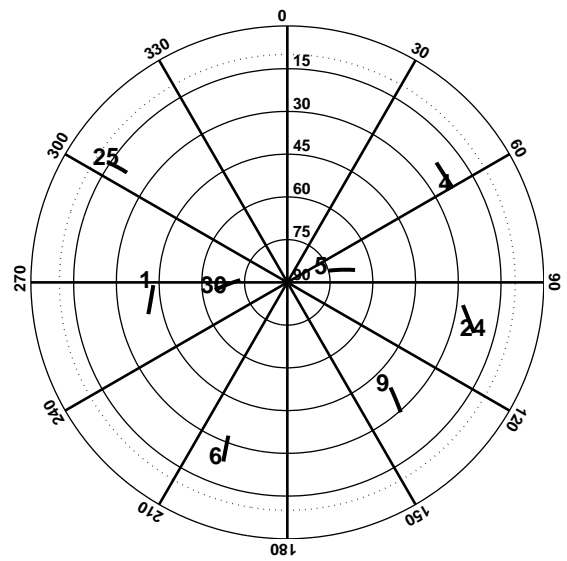

Fig. 1. Receiver-satellite geometry (period: 20 minutes, cut-off elevation: $10^{\circ}$, sampling-interval: 10 seconds) used, with $s=8, s=6$ (PRNs 1,4,5,6,9,24) and $s=4$ (PRNs 1,4,5,6).

that unfortunately still quite long observational periods are needed for the success-rates to become large enough. With 8 satellites and an observational period of 20 minutes, the success-rate is still only at the level of 0.6. The conclusion reads therefore that full ambiguity resolution based on triple-frequency, phase data only can not be expected successful for long baselines when observational periods of shorter than, say, 30 minutes are used. This situation changes however, when the aim is not to resolve all ambiguities, but only a subset of the ambiguities. In this case one speaks of partial ambiguity resolution instead of full ambiguity resolution. The results of partial ambiguity resolution are shown in figure $2 \mathrm{~b}$ for the case one aims at resolving only the $z_{3}$ ambiguities of (14). In this case about $10(s=8)$ to $15(s=6)$ minutes are needed to resolve the ambiguities successfully. These numbers are of course still large when compared to what is already achievable with dual-frequency phase-only data for short baselines. But this is quite understandable and not a fair comparison, since the ionospheric delays are assumed to play a minor role in the models used for short baselines. For long baselines however, one will have to take the ionospheric delays into account. The above results are therefore promising for situations where one rather would do without biasing code data (e.g. stations of permanent networks with high multipath sensitivity). 

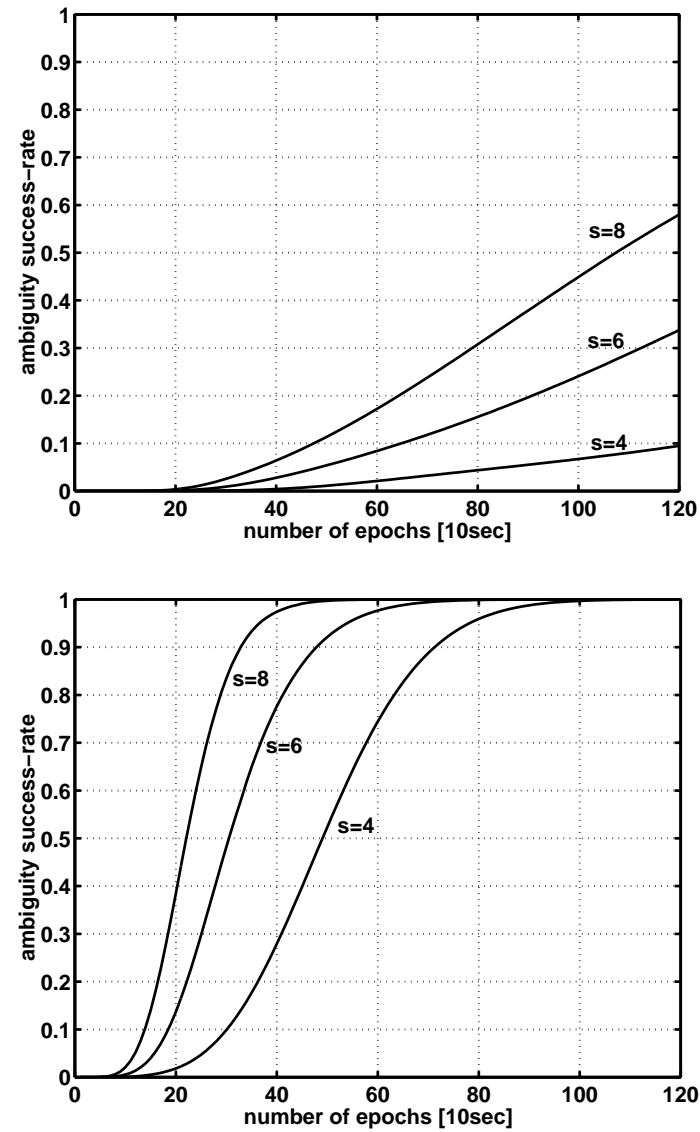

Fig. 2. Ambiguity success-rates as function of the number of satellites tracked and the number of epochs used: (a) resolution of all ambiguities $z_{2}$ and $z_{3}$; (b) resolution of $z_{3}$ ambiguities only.

\section{References}

Hofmann-Wellenhof, B., H. Lichtenegger, J. Collins (1997): Global Positioning System: Theory and Practice. 4th edition. Springer Verlag.

Leick, A. (1995): GPS Satellite Surveying. 2nd edition, John Wiley, New York.

Parkinson, B., J.J. Spilker (eds) (1996): GPS: Theory and Applications, Vols 1 and 2, AIAA, Washington DC.

Strang, G., K. Borre (1997): Linear Algebra, Geodesy, and GPS, Wellesley-Cambridge Press.

Teunissen, P.J.G., A. Kleusberg (eds) (1998): GPS for Geodesy, 2nd enlarged edition, Springer Verlag.

Teunissen, P.J.G. (1995): The least-squares ambiguity decorrelation adjustment: a method for fast GPS integer ambiguity estimation. Journal of Geodesy, 70: 65-82.

Teunissen, P.J.G. (1998): On the integer normal distribution of the GPS ambiguities. Artificial Satellites, Vol. 33, No. 2, pp. 49-64.

Teunissen, P.J.G. (1999): An optimality property of the integer least- squares estimator. Journal of Geodesy, 73: 587-593.
Teunissen, P.J.G., P.J. de Jonge, C.C.J.M. Tiberius (1997): The least- squares ambiguity decorrelation adjustment: its performance on short GPS baselines and short observation spans. Journal of Geodesy, 71: 589-602.

Thomsen, H.F. (2000): Evaluation of upper and lower bounds on the success probability. In: Proc. of ION GPS-2000, Nashville, USA, September 19-22, pp. 183188.

Tiberius, C.C.J.M., P.J. de Jonge (1995): Fast positioning using the LAMBDA method. Proceedings DSNS-95, paper $30,8 \mathrm{p}$. 